\title{
Electron Wave Filters from Inverse Scattering Theory
}

\author{
Daniel Bessist, Giorgio Mantical, G. Andrei Mezincescull and Daniel Vrînceanul \\ Center for Theoretical Study of Physical Systems, Clark - Atlanta University, Atlanta, GA 30314
}

(May 3, 1996)

\begin{abstract}
Semiconductor heterostructures with prescribed energy dependence of the transmittance can be designed by combining: a) Padé approximant reconstruction of the S-matrix; b) inverse scattering theory for Schrö̈inger's equation; c) a unitary transformation which takes into account the variable mass effects. The resultant continuous concentration profile can be digitized into an easily realizable rectangular-wells structure. For illustration, we give the specifications of a 2 narrow band-pass 12 layer $A l_{c} G a_{1-c} A s$ filter with the high energy peak more than twice narrower than the other.
\end{abstract}

85.30D, 03.65N, 73.20D, 73.61D

The inverse scattering method [1 3] allows the reconstruction of the potential, $V(x)$, in the one dimensional Schrödinger equation,

$$
-\frac{\hbar^{2}}{2 m_{0}} \psi^{\prime \prime}+V(x) \psi=E \psi
$$

from the energy dependence of the S-matrix (complex reflection and transmission coefficients). Let us briefly review the necessary results, referring to [3] for details. Setting $E=\hbar^{2} k^{2} / 2 m_{0}>0$, the transmission, $T(k)$, and the left/right reflection coefficients, $R_{ \pm}(k)$ satisfy

$$
\begin{array}{r}
R_{+}(k) T(-k)+R_{-}(-k) T(k)=0, \\
T(k) T(-k)+R_{ \pm}(k) R_{ \pm}(-k)=1, \\
T(k)=[T(-k)]^{*} ; \quad R_{ \pm}(k)=\left[R_{ \pm}(-k)\right]^{*},
\end{array}
$$

where * denotes complex conjugation. In addition, $\left|R_{ \pm}(k)\right|<1$, except at $k=0$, where generically one has $R_{-}(0)=-1$. Moreover, $\lim _{k \rightarrow \infty} R_{ \pm}(k)=0$.

The scattering coefficients $T(k)$ and $R_{ \pm}(k)$ can be continued analytically to the upper half-plane of the complex variable $k$. We will consider only the case when $V(x)=0$ for $x<0$ and there are no bound states. Then, for $\operatorname{Im}(k)>0, T(k)$ and the left-reflection coefficient, $R_{-}(k)$, are analytic functions having no zeros. The rightreflection coefficient, $R_{+}(k)$, may have additional poles. The relations (2,3) remain valid in the whole upper complex half-plane, while eqs. (4) hold with $-k$ replaced by $-k^{*}$ in the right hand sides.

The potential $V(x)$ is reconstructed as

$$
V(x)=\frac{\hbar^{2}}{m_{0}} \frac{\mathrm{d}}{\mathrm{d} x} K_{-}(x, x),
$$

where the function $K_{-}(x, y)$ satisfies Marchenko's integral equation

$$
K_{-}(x, y)+M_{-}(x+y)+\int_{-y}^{x} M_{-}(y+s) K_{-}(x, s) \mathrm{d} s=0 .
$$

Here, $M_{-}(u)$ is the Fourier transform of the (complex) left-reflection coefficient, $R_{-}(k)$, which is therefore the input information required for the inverse technique.

Although inverse scattering methods have been extensively used in various branches of physics and engineering [3, 4], they have not yet been used in heterostructure design. In this Letter we give a brief outline of the application of these techniques to the design of electron-wave filters - semiconductor heterostructures having a prescribed transmittance, $|T(k)|^{2}$. So far, such filters had been designed using methods adapted from optical filter synthesis [5]. These structures may be useful for farinfrared lasers and detectors [6, [].

In the effective mass - envelope function approximation, the electron states in the conduction band of a heterostructure having a position dependent composition $(x$ is the deposition direction and $c(x)$ the alloy concentration) are solutions of the BenDaniel and Duke equation 8 10:

$$
-\nabla \frac{\hbar^{2}}{2 m(x)} \nabla \psi+\mathcal{E}_{c}(x) \psi=E \psi
$$

where $m(x)=m_{\text {eff }}[c(x)]$ and $\mathcal{E}_{c}(x)=\mathcal{E}_{\text {cond }}[c(x)]$ are the local (isotropic) effective mass and conduction band offset of conduction band electrons. We consider here only the case when the device is embedded (at least in an asymptotic sense) in a crystal with uniform composition, i.e. we assume that $c=c_{\infty}$ for $x<0$ and in the limit for $x \longrightarrow+\infty$; let $m_{\infty}$ be the conduction-band effective mass in the embedding material. In many systems of interest, such as $A l_{c} G a_{1-c} A s$, the dependence of $m_{e f f}$ and $\mathcal{E}_{\text {cond }}$ on $c$ is approximately linear [11]: $m_{\text {eff }}(c)=$ $a+b c, \mathcal{E}_{\text {cond }}(c)=\alpha+\beta c$. The mass - concentration dependence can then be inverted, to get:

$$
\mathcal{E}_{c}(m)=\mathcal{A}+\mathcal{B} m \text {. }
$$

For $A l_{c} G a_{1-c} A s$ we use the numerical values $\mathcal{A}=$ $-624.1 \mathrm{meV}$ and $\mathcal{B}=9315 \mathrm{meV} / m_{e}$, where $m_{e}$ is the free electron mass.

After separating away the variables associated with motions orthogonal to the $x$ direction in eq. (7) and setting the transverse quasimomentum $\mathbf{q}_{\perp}=0$, the envelope function for the $x$ direction, $f$, satisfies: 


$$
-\left(\frac{\hbar^{2} f^{\prime}}{2 m}\right)^{\prime}+\mathcal{B}\left[m(x)-m_{\infty}\right] f=\frac{\hbar^{2} k^{2}}{2 m_{\infty}} f .
$$

In the above, we have used (8) and set

$$
\hbar k=\sqrt{2 m_{\infty} E},
$$

the energy $E$ being measured from the conduction band edge of the embedding crystal. An additional term $E_{t}\left(\mathbf{q}_{\perp}\right)\left(\left[m_{\infty} / m(x)-1\right] f(x)\right.$, with $E$ in $(10)$ replaced by $E-E_{t}\left(\mathbf{q}_{\perp}\right)$, where $E_{t}\left(\mathbf{q}_{\perp}\right)=(1 / 2) \hbar^{2} \mathbf{q}_{\perp}^{2} / 2 m_{\infty}$, appears in the left hand side of (9) for $\mathbf{q}_{\perp} \neq 0$. In $A l_{c} G a_{1-c} A s$ the effect of this corrections is quite small for $E_{t}\left(\mathbf{q}_{\perp}\right) \approx k_{B} T$, up to room temperature. Eq. (9) is similar to the one-dimensional Schrödinger equation (11), but with a position-dependent mass. This variability has nontrivial dynamical effects [12]. Noticing that only $m(x)$ enters (9), our task is now to determine mass (and hence concentration $c(x)$ ) profiles giving rise to the pre-assigned transmissivity $|T(k)|^{2}$. Let us highlight the main problems and our approach to solving them.

1. Eq. (7) is a low energy approximation, valid only for the sufficiently small energies at which the conduction band energy is parabolic. Since we assume to be interested only in this range of energies, the transmittance behavior can be arbitrarily continued to higher energies, respecting the analyticity properties of the $S$ matrix. The design problem now has an infinity of solutions. We will not explore all of them, but we will use Padé approximants [13] to fit the transmittance specifications within given tolerances in the required energy range. The non-unicity of the approximants will give free parameters which can be used in successive developments to optimize properties of the device other than the energy dependence of $|T|^{2}$.

2. The transmittance input data we use give only $|T(k)|^{2}$ on the real axis, while the solution of the inverse problem for (1) requires also the phases of $R_{ \pm}$. Generally speaking, the necessary phases can be reconstructed by using dispersion relations as in particle theory 14. Yet, since we use Padé (rational) approximants for fitting the energy dependence of $|T|^{2}$, we can perform the analytic continuation in a simpler way. In fact, in the case when no bound states are present, we let

$$
\mathcal{R}(E)=\frac{\mathcal{P}_{p}(E)}{\mathcal{Q}_{q}(E)},
$$

be the Padé approximant fitted to the reflectance. $\mathcal{P}_{p}(E)$ and $\mathcal{Q}_{q}(E)$ are polynomials of degree $p$ and $q \geq p+2$ with real coefficients. The condition $q \geq p+2$ guarantees that $\mathcal{R}(E)=\mathcal{O}\left(E^{-2}\right)$ for large $E$ and, therefore, the potential is non-singular at $x=0$. In accordance with the analytic properties of the reflectance on the real axis, (2) (1), $0 \leq \mathcal{R}(E)<1$ for $0<E<\infty$ and $\mathcal{R}(0)=1$. The latter implies that $\mathcal{P}_{p}(0)=\mathcal{Q}_{q}(0)=1$. From the former follows that if $\mathcal{P}_{p}(E)$ has real positive zeros (which correspond to resonances, where the transmittance attains its maximal allowed value, unity) their multiplicities have to be even. From the structure of eq. (6), we see that it is convenient to work in $k$ space, and to compute $(p, q)$ Padé approximants to the left-reflection coefficient

$$
R_{-}(k)=\frac{P_{p}(k)}{Q_{q}(k)},
$$

where the $k=0$ values of the polynomials are taken as $P_{p}(0)=-Q_{q}(0)=-1$. This guarantees that $R_{-}(0)=$ -1 . Let $k_{\alpha}$ (respectively, $k_{j}$ ) be the zeros of $P(Q)$. To satisfy the analytic properties of $R_{-}(k)$ and the condition (4), the zeros of $P$ and $Q$ must have non-positive imaginary parts (negative for $P$ ). If $k_{\alpha}\left(k_{j}\right)$ is a root of $P(k)=0(Q(k)=0)$, then $-k_{\alpha}^{*}\left(-k_{j}^{*}\right)$ must also be a zero of the same polynomial: zeros of $P$ and $Q$ which are not purely imaginary come in pairs. Using the root product expansion of the polynomials we may write $R_{-}(k)=-\prod_{\alpha=1}^{p}\left(1-\frac{k}{k_{\alpha}}\right) / \prod_{j=1}^{p}\left(1-\frac{k}{k_{j}}\right)$. For real positive energies the reflectance is given by

$$
\mathcal{R}(E)=R_{-}(k) R_{-}(-k),
$$

where we used (4). Thus, taking into account (10), we see that the zeros and poles of the analytic continuation to the complex $E$ plane of the Padé approximant (11) for the real axis reflectance are connected to the zeros and poles of the Padé approximant (12) for the (complex) reflection coefficient by $k= \pm \sqrt{2 m_{\infty} E} / \hbar$. The sign assignments are unambiguous in the absence of bound states, when $R_{-}(k)$ is analytic and has no zeros in the upper half plane. The phase reconstruction for the analytic continuation of the reflection coefficient $R_{-}(k)$ from the absolute values on the real axis is therefore achieved.

Similarly, we seek the transmission coefficient, as a $(q, q)$ Padé approximant having the same poles as $R_{-}(k)$ :

$$
T(k)=\frac{k L_{q-1}(k)}{Q_{q}(k)} .
$$

Here we used $T(0)=0$. The polynomial $L_{q-1}(k)$ is determined by its roots and the condition $\lim _{k \rightarrow \infty} T(k)=1$. From (13) and (3,4), the transmittance on the real axis is given by $\mathcal{T}(E)=1-R_{-}(k) R_{-}(-k)$ and (14) leads to the following equation for the zeros of $L_{q-1}(k)$

$$
k^{2} L_{q-1}(k) L_{q-1}(-k)=\mathcal{Q}_{q}(E)-\mathcal{P}_{p}(E)=0 .
$$

The zeros of $L_{q-1}(k)$ are then given via (10) (taken with non positive imaginary part) by the zeros of the analytic continuation in energy of the absolute value of $\mathcal{T}(E)$. Finally, $R_{+}(k)$ can be obtained from (2) and we have fully reconstructed the S-matrix from the Padé approximation for the energy dependence of the transmittance, in the absence of bound states. 
3. Although similar, (9) differs from (11) by having a variable unknown effective mass.

In $A l_{c} G a_{1-c} A s, m_{e f f}$ increases by about $50 \%$ from the $G a A s$ value in the direct gap concentration range, $0 \leq c<0.45$. There are two alternative ways to tackle this problem. One may reformulate the inverse scattering method to deal with equation (9). We prefer to follow an alternative, two-step approach. First, we solve an auxiliary problem: we find a potential for the constant mass Schrödinger equation (11) such that the corresponding $S$-matrix coincides with the one reconstructed from the transmittance, as in point 2 above. In particular, if the scattering data are expressed by Padé approximants, then the solution of the inverse problem is much simplified, and it can be accomplished by solving a linear system of equations [1 3]. Second, we will use a unitary transformation linking constant and variable mass Hamiltonians 15 and derive a differential equation for the mass (concentration) profile in (9).

In fact, let $X(\xi)$ be a twice differentiable function, satisfying $X( \pm \infty)= \pm \infty$ and having a positive derivative $\frac{\mathrm{d} X}{\mathrm{~d} \xi}=\dot{X}(\xi)>0$. Here and in the following we use the dot to denote derivation with respect to $\xi$. Then, let us consider the coordinate transformation

$$
x=X(\xi),
$$

which maps the interval $(-\infty,+\infty)$ onto itself. As shown in 15], each such change of variables corresponds to a unitary operator, $U_{X}$, in the space of square integrable functions, such that in the new coordinate $\xi$ the equation (11) transforms to

$$
-\frac{\mathrm{d}}{\mathrm{d} \xi} \frac{\hbar^{2}}{2 m(\xi)} \frac{\mathrm{d}}{\mathrm{d} \xi} f(\xi)+V_{X}(\xi) f(\xi)=E f(\xi)
$$

where

$$
\begin{gathered}
m(\xi)=m_{0} \dot{X}^{2} \\
V_{X}(\xi)=V[X(\xi)]+\frac{\hbar^{2}}{8 m_{0} \dot{X}^{4}}\left(2 \dddot{X} \dot{X}-5 \ddot{X}^{2}\right),
\end{gathered}
$$

and where we omitted for simplicity the $\xi$ dependence of the derivatives of $X$. Eqs. (17-19) and (9) coincide if

$$
V_{X}(\xi)=\mathcal{B}\left[m(\xi)-m_{\infty}\right],
$$

where $m(\xi)$ and $V_{X}(\xi)$ are given by $(18,19)$. This is a nonlinear differential equation of third order for $X$. Noting that it does not depend explicitly on $\xi$, and introducing a new dependent variable $S(X)=\dot{X}$ yields

$$
2 S^{\prime \prime} S-3 S^{2}=\frac{8 m_{0} S^{2}}{\hbar^{2}}\left[\mathcal{B} m_{0}\left(S^{2}-S_{\infty}^{2}\right)-V(X)\right],
$$

The prime denotes derivation with respect to $X$.
To be physically feasible, the solution of (21) must be regular and bounded by

$$
\underline{m} \leq m_{0} S^{2}(\xi) \leq \bar{m}
$$

where $\underline{m}$ and $\bar{m}$ are the minimal and maximal achievable values of the effective mass, and tend to $\sqrt{m_{\infty} / m_{0}}$ in the limits $X \rightarrow \pm \infty$. A necessary condition for the latter condition to hold is $\mathcal{B}>0$, which is validated for GaAlAs. The asymptotic value of the effective mass for the embedding crystal is a free parameter. Its value must be chosen in a range leading to a feasible concentration range, satisfying (22).

In practice, we truncate the auxiliary (constant mass) potential $V(X)$ to a finite interval $(0, L)$. For zero potential Eq. (21) can be explicitly solved: $S(X)=$ $\left[a+b \mathrm{e}^{\kappa X}+c \mathrm{e}^{-\kappa X}\right]^{-1}$, where $\hbar^{2} \kappa^{2}=8 \mathcal{B} m_{\infty}$ and the constants $a, b, c$ satisfy $a^{2}-4 b c=m_{0} / m_{\infty}$. Now, let $S(X)$ be a regular solution on one of the intervals $(-\infty, 0)$ or $(L,+\infty)$. It satisfies

$$
S^{\prime}(X)= \pm \kappa S(X)\left[1-\sqrt{m_{0} / m_{\infty}} S(X)\right]
$$

at all points of the respective interval, with the minus sign on the former and the plus on the latter. Thus, in order to be continuous and achievable, a solution of (21) on $(0, L)$ must satisfy (23) at the ends of the interval. This is a nonlinear Sturm-Liouville problem that can be solved by the shooting method. The new coordinate can be obtained then by integrating $\dot{X}(\xi)=S(X)$. Reasonable precision may also be obtaineded by using a quasi-classical approximate solution to (21).

Thus, we obtain a continuous distribution of mass (concentration) yielding the desired transmittance. Technological constraints make desirable concentration distributions in the form of a sequence of steps of digitized length (in lattice constants) and height (linear combinations of several concentrations). This can be achieved by approximating the continuous concentration profile and by using standard optimization techniques. The brute force approach to the inverse problem is to find the transfer matrix of (9) for concentration profiles made of discrete steps (parameterized by concentrations and lengths) and constrain the resultant transmittance to the predetermined profile. This suffers from the fact that the corresponding optimization problem is non-convex and, even using the largest computers, satisfactory results are not guaranteed. To the contrary, our approach offers a good starting point for the optimization process. A modest exploration of its neighborhood usually provides superb results.

As an illustration, we give in Table the data for a 12 layer $A l_{c} G a_{1-c} A s$ filter with digitized steps in width and concentration. Quite remarkably, this structure accomplishes the goal of having a lower energy resonance more than twice wider than the high-energy one. In Fig. 
1 the transmittance computed from (9) is compared to a constant effective mass approximation using (1) with $m_{0}=m_{\infty}$. The latter's errors become important at the higher resonance, whose width is overestimated by a factor of two, thereby losing the main feature of this structure! The computation of the continuous profile takes several seconds on an IBM RISC 6000 workstation. The digitizatization and final optimization were done manually.

Spatial variations of the band gap have been crucial for the success of man-made heterostructures. We have outlined a technique which takes into account also the spatial variations of effective mass, and can be employed in the design of various electronic devices.

Acknowledgment. This research supported in part by NSF and the CAU Center for Environmental Policy, Education and Research under EPA Assistance ID \# CR818689. We thank Thomas K. Gaylord and Elias Glytsis for bringing this problem to our attention together with a wealth of information on semiconductor heterostructure design. We thank Roger Balian, Khosrow Chadan and Pierre Sabatier for stimulating discussions on inverse scattering.

* $\quad$ Service de Physique Théorique, C.E. Saclay, 91191 Gif sur - Yvette Cedex, France

$\S \quad$ Istituto di Scienze Matematiche e Fisiche, Università di Milano sede di Como, Via Lucini 3, 22100 Como, Italy

$\dagger \quad$ Institutul de Fizica şi Tehnologia Materialelor, C.P. MG - 7, Bucureşti - Măgurele, Romania

¥ Facultatea de Fizică, Universitatea Bucureşti, C.P. MG - 11, Bucureşti - Măgurele, Romania

[1] I. Kay and H. Moses, Nuovo Cimento 3, 276 (1955).

[2] P. Sabatier, Nuovo Cimento 78B, 235 (1983).

[3] K. Chadan and P. C. Sabatier, Inverse Problems in Quantum Scattering Theory, 2 ed. (Springer, Berlin, 1989).

[4] P. P. Roberts and G. E. Town, IEEE Trans. Microwave Th. and Techn. 43, 739 (1995).

[5] T. Gaylord, E. Glytsis, and K. Brennan, J. Appl. Phys. 65, 2535 (1989); G. N. Henderson, T. K. Gaylord, and E. N. Glytsis, Phys. Rev. B 45, 8404 (1992).

[6] S. I. Borenstain and J. Katz, Appl. Phys. Lett. 55, 654 (1989); M. Helm and S.J. Allen jr., Appl. Phys. Lett. 56, 1368 (1990); Q. Hu and S. Feng, Appl. Phys. Lett. 59, 2923 (1991).

[7] J. Feist and et. al., Science 264, 553 (1994).

[8] D. J. BenDaniel and C. B. Duke, Phys. Rev. 152, 683 (1966).

[9] G. Bastard, Wave mechanics applied to semiconductor heterostructures (Editions de Physique, Les Ulis, France, 1988).

[10] M. R. Geller and W. Kohn, Phys. Rev. Lett. 70, 3103
(1993).

[11] R. Kopf, M. Herman, M. Schnoes, and C. Colvard, J. Vac. Sci. Technol. B 11, 813 (1993),

[12] A rigorous treatment in the case of periodic alloy concentration $c(x)$ may be found in: G. Mantica and S. Mantica, Phys. Rev. B 46, 7037 (1992).

[13] G. Baker jr, Essentials of Padé Approximants (Academic Press, New York, 1975),

[14] R. Omnes, Nuovo Cimento 8, 317 (1958); D. Bessis, Calculation of the phase of transmission coefficient using finite order dispersion relations (to be published).

[15] R. Balian, D. Bessis, and G. A. Mezincescu, Phys. Rev. B 51, 17624 (1995).

TABLE I. 12 layer digitized $A l_{c} G a_{1-c} A s$ filter.

\begin{tabular}{cccc}
\hline $\begin{array}{c}\text { Layer } \\
\#\end{array}$ & $\begin{array}{c}\text { Width } \\
\left(\text { unit }^{\mathrm{a}}\right)\end{array}$ & $\begin{array}{c}\text { Width } \\
(\mathrm{nm})\end{array}$ & $\begin{array}{c}\text { Al } \\
\text { concentration }\end{array}$ \\
\hline 1 & 6 & 1.696 & $c_{1}+c_{2}+c_{3}$ \\
2 & 9 & 2.543 & 0 \\
3 & 18 & 5.088 & $c_{3}$ \\
4 & 5 & 1.413 & $c_{2}$ \\
5 & 10 & 2.827 & $c_{1}+c_{2}$ \\
6 & 10 & 2.827 & $c_{1}$ \\
7 & 14 & 3.957 & $c_{1}+c_{2}$ \\
8 & 14 & 3.957 & $c_{1}$ \\
9 & 13 & 3.675 & $c_{1}+c_{2}$ \\
10 & 13 & 3.675 & $c_{1}$ \\
11 & 14 & 3.957 & $c_{1}+c_{2}$ \\
12 & 11 & 3.109 & $c 1$ \\
\hline \hline
\end{tabular}

${ }^{\mathrm{a}}$ monoatomic layer width $=0.2827 \mathrm{~nm}$

${ }^{\mathrm{b}} c_{1}=0.05714, c_{2}=2 c_{1}, c_{3}=4 c_{1}$, bulk concentration $=c_{2}$

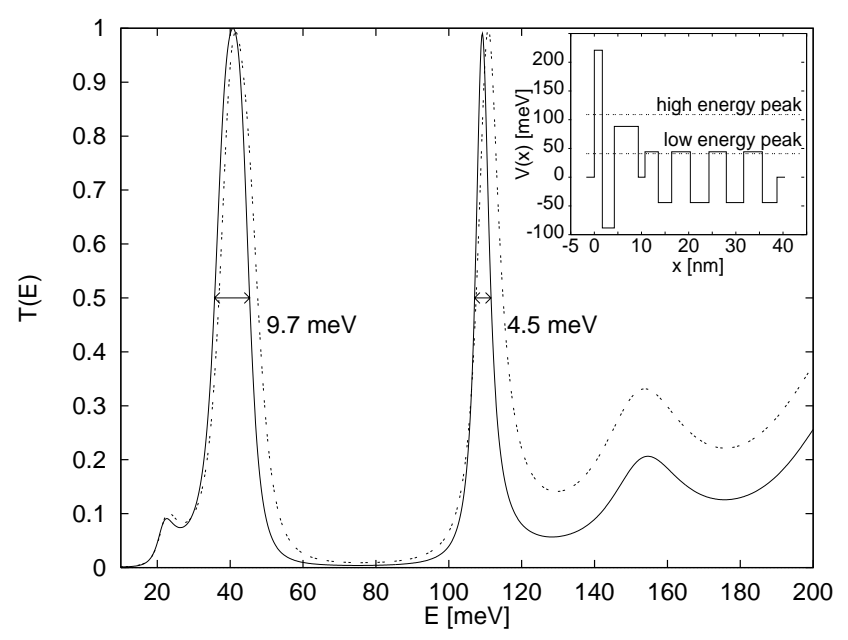

FIG. 1. Energy dependence of the transmittance of 12 layer digitized $A l_{c} G a_{1-c} A s$ filter: continuous line - Eq.(9); dotted line - constant mass approximation Eq.(1) with $m_{0}=m_{\infty}$. Insert: potential energy profile. 